PCR procedure. Only a fraction of all pyrimidines within a DNA strand form dimers and, as dimers are both made and broken by ultraviolet exposure, this fraction establishes a steady-state level which varies with the irradiation wavelength, the type of pyrimidine dimer and the nucleotide sequences next to the dimer site $^{2.3}$. At steady-state, an upper limit for the number of all dimer defects in a long, irradiated DNA molecule is less than 0.065 defects per base pair 4 . Non-dimer photodamage (for example noncyclobutane-type pyrimidine adducts, thymine glycols, interstrand and intrastrand DNA-DNA crosslinks and DNA strand breaks) can also be termination sites for Taq DNA polymerase. The number of these sites should be at least equivalent to the number of dimer sitess.

If these defects $(0.13$ total defects per base pair) are randomly distributed throughout a DNA molecule, a 500base-long oligonucleotide would have an average of 32 damaged sites. In a population of $10^{5}$ DNA strands containing an average of 32 termination sites per strand, there is a minute probability that any strand is without at least one termination site. By comparison, 100-base-long PCR products will have an average of only 6 modified sites per strand at this same defect density. In a population of $10^{5}$ molecules with just six average termination sites per strand, a statistically large number of molecules will contain no modified sites and therefore are not sterilized.

Furthermore, to be effective for PCR sterilization, the photoinduced defects should be in the sequence region bounded by the $3^{\prime}$ ends of the PCR primers. These considerations predict that ultraviolet irradiation would be effective for long but not for short PCR products. Our experiments demonstrate that this is the case.

We irradiated targets with either 254 , 300 or a combination of 254 and $300-\mathrm{nm}$ bulbs. After irradiation, the remaining PCR reaction components were added to each tube and amplification was performed. As shown in the figure, a $115 \mathrm{mer}$ PCR product is efficiently generated from as little as $10^{3}$ target molecules that had been irradiated with a combined 254 and $300 \mathrm{~nm}$ exposure independent of whether the target is a synthetic 115 mer or an $11-\mathrm{kb}$ plasmid. Irradiation of linerized $\lambda$ DNA from which a $500 \mathrm{mer}$ PCR product is made results in $10^{3}$ copies of the $\lambda$ being sterilized with the combined $254 / 300 \mathrm{~nm}$ procedure. But $10^{5}$ copies of the $\lambda$-DNA target show an exposure dependent sterilization. Only 30 minutes of exposure is sufficient to reduce the PCR signal below the sensitivity level of the gel assay.

These data imply that caution is needed when applying direct ultraviolet irradiation as a sterilization process in a preamplification mode. Both the length of the PCR product and its internal sequence must be considered. The 115 mer we used was chosen because it has a very high fraction of adjacent thymines between the primer regions of the PCR product. But the distribution of thymines is such that most of the potential pyrimidine dimers will occur on one strand of the product. This type of distribution must also be taken into account when considering ultraviolet sterilization techniques.

GEORGE D. CIMINO

KENNETH METCHETTE

STEPHENT. ISAACS

YU SHENG ZHU

HRI Research Inc,

Berkeley, California 94710, USA

1. Sarkar, G. \& Sommer, S. Nature 343, 27 (1990)

2. Setlow, R. B. \& Setlow, J. K. Proc. natn. Acad. Sci. U.S.A 48, $1250-1257$ (1962).

Gordon, L. K. \& Haseltine, W. A. Rad. Res, 89, 99-112 (1982)

. Rahn, R. O. in Photophysiology (ed. Grise, A. C.) 231-255 (Academic, New York, 1973)

Rahn, R. O. Photochem. Photobiol. Rev. 4, 267-330 (1979)

6. Ou, C-Y, et al. Science 239, 295-297 (1988)

\section{Hiccup for hiccups}

SIR-Fuller' suggests that hiccups represent an essential intrauterine reflex to allow vigorous exercise of fetal inspiratory musculature in preparation for extrauterine breathing. But this idea has not been seriously advanced since 1902 . Respiratory views of hiccup function fell from favour as gastrointestinal rather than respiratory events were found to promote hiccups (for example, ref. 2).

Contrary to Fuller's claim, hiccups differ from developmental reflexes in that they do not entail reliable muscle or nerve actions (one or both hemidiaphragms may be involved, or accessory muscles of respiration, and possible afferents are legion); they are never reliably elicited; and they do not cease at a defined developmental stage. The argument that hiccups affect all humans and respect a developmental profile is equally valid for bedwetting and drooling. These have no function but reflect immaturity - or pathological disruption - of neuronal control systems. Even cardiac arrhythmias are universal. Arrhythmias, like hiccups, may result from an imbalance of neuronal excitation and inhibition. Indeed, both heart block and hiccups are provoked by high vagal tone, and the two may occur together ${ }^{3.4}$. So hiccups could be good for nothing.

The Salk Institute,

B. GoLomB

10010 North Torrey Pines Rd,

La Jolla,

California 92037, USA

1. Fuller, G. N. Nature 343, 429 (1990)

2. Davis, J. N. Brain 93, 851-872(1970)

3. Thorne, M. G. Br. Heart J. 3, 387 (1969)

4. Harrington, J. T. \& De Sanctis, R. W. Ann. Intern. Med. 70 105-106 (1969)

\section{Treat with care}

SIR-The association recently found by Gardner et al. ${ }^{1}$ between paternal exposure to ionizing radiation before conception and childhood leukaemia in offspring has raised considerable concern and pressure to confirm or deny the association. But radiation is only one of the possible risk factors to which men working in an industrial/chemical environment such as that of nuclear-fuel reprocessing might be exposed. Regrettably, the current state of understanding of the underlying theoretical basis for the action of ionizing radiation on living organisms does not allow opinion to be expressed one way or the other on the validity of a causal association with radiation.

Evans, in his News and Views article ${ }^{2}$, has outlined in some detail the reasons for scepticism concerning the possibility that the association reported by Gardner et al. is casual. And in Scientific Correspondence last week ${ }^{3}$, Nomura, whose experiments with mice ${ }^{4}$ have been widely quoted, pointed out the factors that may confound extrapolation of his results to help explain a causal basis for the data of Gardner et al.

Childhood leukaemia is not necessarily related to any form of leukaemia in adulthood, and probably has origins at the embryo stage, as shown by the increased risk of this disease in children irradiated in utero ${ }^{5}$. In mice, no distinction can be made between such neoplastic disease and adult neoplasia, and there is no authenticated model for human childhood leukaemia in any animal.

For these reasons, and also because of the marked strain differences in radiationinduced neoplasia in animals and the scale of experiments required to detect effects at low doses and low-dose rates, it would be extremely difficult to devise a reliable test of the Gardner hypothesis using animals.

But Gardner et al. have put forward a clear-cut hypothesis relating doses before conception to a specified increase in the risk of childhood leukaemia. Although this is obviously not a simple task, the hypothesis should be tested on other populations of workers who are exposed to radiation but not to the other hazards of chemical processing, such as organic solvents (some of which are known to be leukaemogens), before people begin large-scale animal experimentation.

$$
\text { K. F. BAVERSTOCK }
$$
E. G. WRIGHT

MRC Radiobiology Unit, Chilton, Didcot, Oxon OX1 ORD, UK

\footnotetext{
Gardner, M.J. et al. Br. med. J. 300, 423-434 (1990).

2. Evans, H.J. Nature 345, 16-17 (1990)

3. Nomura, T. Nature 345, 671 (1990)

4. Nomura, T. Nature 298, 575-577 (1982)

5. Bithell, J.F. in Low Dose Radiation: Biological Bases of Risk Assessment (eds Baverstock, K.F. \& Stather, J.W.) 77-87 (Taylor \& Francis, London, 1989).
} 